Modelling, Analysis and Simulation

Modelling, Analysis and Simulation
MAS Two new variants of the manifold-mapping technique

D. Echeverría 
Centrum voor Wiskunde en Informatica (CWI) is the national research institute for Mathematics and Computer Science. It is sponsored by the Netherlands Organisation for Scientific Research (NWO).

CWI is a founding member of ERCIM, the European Research Consortium for Informatics and Mathematics.

CWI's research has a theme-oriented structure and is grouped into four clusters. Listed below are the names of the clusters and in parentheses their acronyms.

Probability, Networks and Algorithms (PNA)

Software Engineering (SEN)

\section{Modelling, Analysis and Simulation (MAS)}

Information Systems (INS)

Copyright (C) 2006, Stichting Centrum voor Wiskunde en Informatica

P.O. Box 94079, 1090 GB Amsterdam (NL)

Kruislaan 413, 1098 SJ Amsterdam (NL)

Telephone +31205929333

Telefax +31205924199

ISSN 1386-3703 


\title{
Two new variants of the manifold-mapping technique
}

\author{
ABSTRACT \\ Manifold-mapping is an efficient surrogate-based optimization technique aimed at the \\ acceleration of very time-consuming design problems. In this paper we present two new \\ variants of the original algorithm that make it applicable to a broader range of optimization \\ scenarios. The first variant is useful when the optimization constraints are expressed by means \\ of functions that are very expensive to compute. The second variant endows the original \\ scheme with a trust-region strategy and the result is a much more robust algorithm. By two \\ practical design problems from electromagnetics we eventually show that the proposed variants \\ perform efficiently.
}

2000 Mathematics Subject Classification: 90C30, 65K05, 49M99, 49Q99

Keywords and Phrases: multi-level optimization, surrogate optimization, trust-region methods, space mapping, manifold mapping, electromagnetic devices design

Note: This research is supported by the Dutch Ministry of Economic Affairs through the project IOP-EMVT-02201 B. 



\title{
Two New Variants of the Manifold-Mapping Technique
}

\author{
D. Echeverría* \\ Centrum voor Wiskunde en Informatica (CWI) \\ Kruislaan 413, 1090 GB, Amsterdam, The Netherlands
}

\begin{abstract}
Manifold-mapping is an efficient surrogate-based optimization technique aimed at the acceleration of very time-consuming design problems. In this paper we present two new variants of the original algorithm that make it applicable to a broader range of optimization scenarios. The first variant is useful when the optimization constraints are expressed by means of functions that are very expensive to compute. The second variant endows the original scheme with a trust-region strategy and the result is a much more robust algorithm. By two practical design problems from electromagnetics we eventually show that the proposed variants perform efficiently.
\end{abstract}

Keywords: multi-level optimization, surrogate optimization, trustregion methods, space-mapping, manifold-mapping, electromagnetic devices design.

\section{Introduction}

Space mapping (Bandler et al., 1994, 2004; Echeverría et al., 2005) was introduced by J.W. Bandler as a surrogate-based optimization technique aimed at optimization processes where very accurate (and quite often time-consuming) models are needed. In space-mapping terminology these models are called fine models. The surrogate is a correction of a fast-to-compute approximation of the fine model. This correction and the approximation are commonly known as the space-mapping function and as the coarse model respectively.

However, the space-mapping approach does not in general yield the correct optimum. Moreover, the computation of the space-mapping function requires an additional optimization problem. This minimization process is sometimes problematic (Bandler et al., 2004). By manifold mapping (Echeverría and Hemker, 2005) we overcome those difficulties and we obtain an improved and efficient optimization strategy.

The manifold-mapping concept introduces an alternative correction of the coarse model. Unlike the space-mapping function, it is very straightforward

*Phone: +31 20592 4122, E-mail: D.Echeverria@cwi.nl. 
to compute and the associated manifold-mapping algorithm can be proven to converge to the true optimum (Echeverría and Hemker, 2006). The manifoldmapping algorithm has been reported as an efficient optimization technique in problems of practical relevance (Echeverría et al., 2006).

In this paper we present two significant improvements of the original manifold-mapping scheme. The first one allows its use in design scenarios where optimization constraints are expressed by functions that are very expensive to compute. The second improvement endows the original iteration with a trustregion strategy that increases the algorithm robustness, when for example, the optimization problem is strongly nonlinear or ill-conditioned (i.e., multiple optima are present).

This paper is structured as follows. In Section 2 the basics of manifold mapping are explained. The two new variants, constrained optimization with manifold mapping and trust-region manifold mapping, are introduced in Section 3. Eventually in Section 4, these two new algorithms are applied to practical design problems from electromagnetics.

\section{Manifold-Mapping Basics}

Before explaining the basics of the manifold-mapping approach, we introduce the notation adopted. The design specifications are denoted by $\mathbf{y} \in \mathbb{R}^{m}$. The fine model response $\mathbf{f}(\mathbf{x})$ is defined over the set $X \subset \mathbb{R}^{n}$ and $\mathbf{x} \in X$ is the design variable. In this work we minimize the discrepancy between the specifications and the fine model response

$$
\mathbf{x}_{\mathbf{f}}^{*}=\operatorname{argmin}_{\mathbf{x} \in X}\|\mathbf{f}(\mathbf{x})-\mathbf{y}\|
$$

We refer to $\mathbf{x}_{\mathbf{f}}^{*}$ as the fine model optimum. Not every optimization problem is of this type, but most practical design situations can be reduced to this structure.

The coarse model response is denoted by $\mathbf{c}(\mathbf{x})$ and it is also defined over $X$. We can consider coarse models over sets $Z \neq X$ by the introduction of an additional mapping $\overline{\mathbf{p}}: X \rightarrow Z$, as in (Echeverría and Hemker, 2005). The coarse model optimum is defined as

$$
\mathbf{x}_{\mathbf{c}}^{*}=\operatorname{argmin}_{\mathbf{x} \in X}\|\mathbf{c}(\mathbf{x})-\mathbf{y}\|
$$

A general constrained optimization problem can be stated with the help of a constraint function $\mathbf{k}_{\mathbf{f}}(\mathbf{x})$ as

$$
\begin{aligned}
\mathbf{x}_{\mathbf{f}}^{*} & =\operatorname{argmin}_{\mathbf{x} \in X}\|\mathbf{f}(\mathbf{x})-\mathbf{y}\| \\
X & =\left\{\mathbf{x} \in \hat{X} ; \mathbf{k}_{\mathbf{f}}(\mathbf{x}) \geq 0\right\},
\end{aligned}
$$

or equivalently

$$
\mathbf{x}_{\mathbf{f}}^{*}=\operatorname{argmin}_{\mathbf{x} \in \hat{X}}\|\mathbf{f}(\mathbf{x})-\mathbf{y}\| \quad \text { subject to } \quad \mathbf{k}_{\mathbf{f}}(\mathbf{x}) \geq 0 .
$$

Now $\mathbf{f}$ and $\mathbf{k}_{\mathbf{f}}$ are defined over $\hat{X} \subset \mathbb{R}^{n}$, a superset of $X$. The function $\mathbf{k}_{\mathbf{f}}$ refers to the fine (accurate) equality and inequality constraints. In this section $\mathbf{k}_{\mathbf{f}}$ will be assumed easy-to-compute and thus, no special care with respect to the constraints has to be taken in the manifold-mapping approach. For those cases 

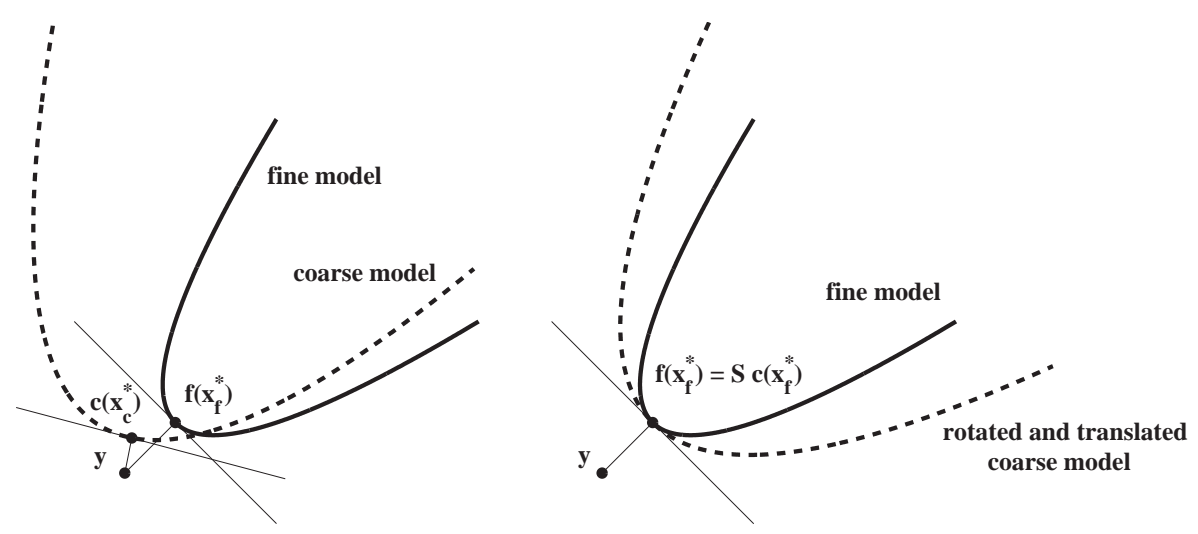

Figure 1: Manifold-mapping model alignment.

where $\mathbf{k}_{\mathbf{f}}$ requires a significant computational effort, manifold mapping can still be applied whenever a fast-to-evaluate function $\mathbf{k}_{\mathbf{c}}$ is available for approximating the fine constraint function $\mathbf{k}_{\mathbf{f}}$ (see Section 3.1).

In (Echeverría and Hemker, 2005) the manifold mapping $\mathbf{S}: \mathbf{c}(X) \rightarrow \mathbf{f}(X)$ is introduced with the aim of correcting the misalignment between the models $\mathbf{f}$ and $\mathbf{c}$. With $\mathbf{S}$, the point $\mathbf{c}\left(\mathbf{x}_{\mathbf{f}}^{*}\right)$ is mapped to $\mathbf{f}\left(\mathbf{x}_{\mathbf{f}}^{*}\right)$ and the tangent plane for $\mathbf{c}(X)$ at $\mathbf{c}\left(\mathbf{x}_{\mathbf{f}}^{*}\right)$ to the tangent plane for $\mathbf{f}(X)$ at $\mathbf{f}\left(\mathbf{x}_{\mathbf{f}}^{*}\right)$ (see Figure 1). Other approaches are possible but in our work we define $\mathbf{S}$ as the affine mapping

$$
\mathbf{S} \mathbf{c}(\mathbf{x})=\mathbf{f}\left(\mathbf{x}_{\mathbf{f}}^{*}\right)+\bar{S}\left(\mathbf{c}(\mathbf{x})-\mathbf{c}\left(\mathbf{x}_{\mathbf{f}}^{*}\right)\right)
$$

where

$$
\bar{S}=J_{\mathbf{f}}\left(\mathbf{x}_{\mathbf{f}}^{*}\right) J_{\mathbf{c}}^{\dagger}\left(\mathbf{x}_{\mathbf{f}}^{*}\right) .
$$

The ${ }^{\dagger}$ symbol represents the pseudoinverse and $J_{\mathbf{f}}\left(\mathbf{x}_{\mathbf{f}}^{*}\right)$ and $J_{\mathbf{c}}\left(\mathbf{x}_{\mathbf{f}}^{*}\right)$ denote respectively the Jacobian of $\mathbf{f}$ and $\mathbf{c}$ at $\mathbf{x}_{\mathbf{f}}^{*}$. The combination $\mathbf{S} \circ \mathbf{c}$ acts as the surrogate model for $\mathbf{f}$. Under some assumptions about the similarity between the fine and the coarse model that usually hold in practice, it can be shown (Echeverría and Hemker, 2006) that

$$
\mathbf{x}_{\mathbf{f}}^{*}=\operatorname{argmin}_{\mathbf{x} \in X}\|\mathbf{S} \mathbf{c}(\mathbf{x})-\mathbf{y}\|=\operatorname{argmin}_{\mathbf{x} \in X}\left\|\mathbf{c}(\mathbf{x})-\mathbf{c}\left(\mathbf{x}_{\mathbf{f}}^{*}\right)+\bar{S}\left(\mathbf{f}\left(\mathbf{x}_{\mathbf{f}}^{*}\right)-\mathbf{y}\right)\right\| .
$$

The last equality is the basis for the manifold-mapping algorithm.

The mapping $\mathbf{S}$ is not known a priori, because it depends on the solution of the optimization problem. But it can be iteratively approximated. The manifold-mapping algorithm (see Figure 2) yields both the mapping $\mathbf{S}$ and the desired fine model optimum $\mathbf{x}_{f}^{*}$ (Echeverría and Hemker, 2006). This scheme is particularly attractive from an implementational point of view: the central function in every minimization process is always the coarse model $\mathbf{c}(\mathbf{x})$ and not a surrogate (the specifications are updated in each step).

\section{Two New Manifold-Mapping Variants}

The manifold-mapping algorithm (MM, Figure 2) in the previous section cannot be applied efficiently when the constraint function $\mathbf{k}_{\mathbf{f}}$ is expensive to compute. 


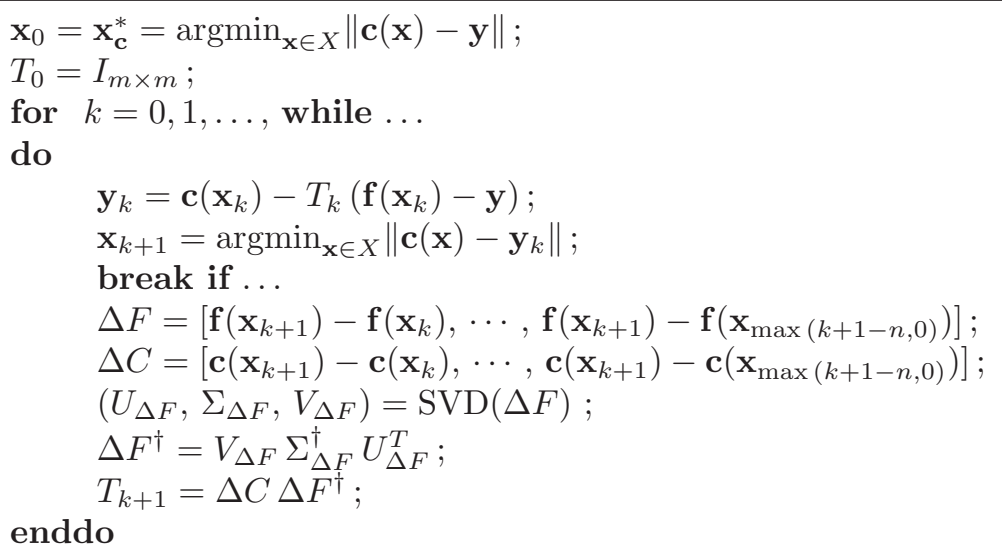

Figure 2: The manifold-mapping (MM) algorithm. SVD denotes a singular value decomposition.

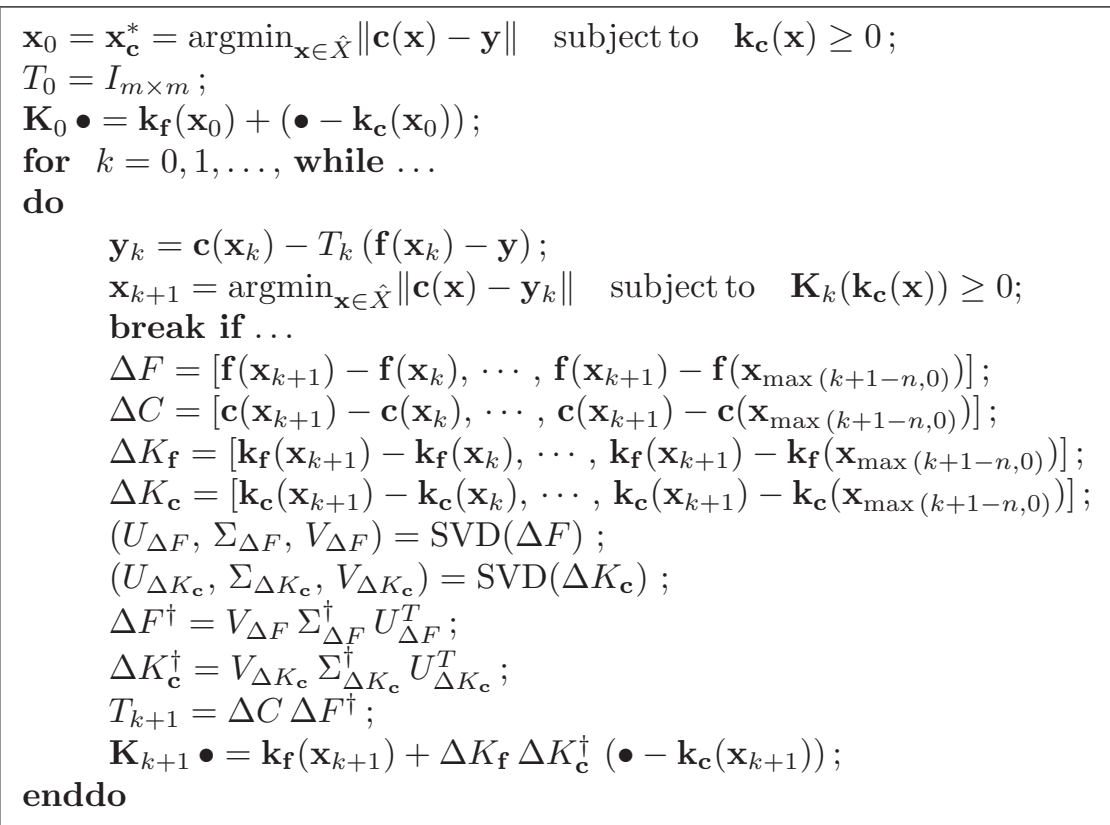

Figure 3: The manifold-mapping algorithm for constrained optimization. SVD denotes a singular value decomposition.

Moreover, it can fail when the models are ill-conditioned, i.e., a small variation of the design variable does not affect the model response. Therefore, we present two new variants of the basic scheme in order to cope with these two problematic situations. 


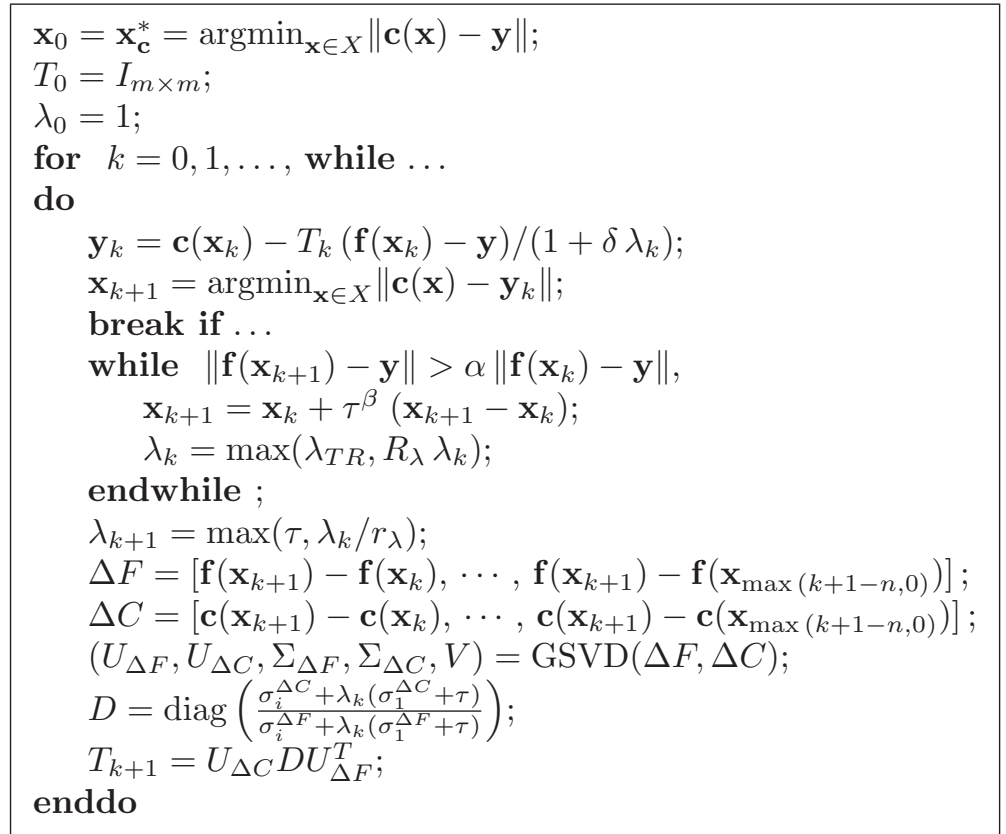

Figure 4: The Trust-Region Manifold-Mapping (TRMM) algorithm. GSVD denotes a generalized singular value decomposition. Typical values for the parameters are: $\delta=0, \alpha=1+\tau>1, \beta=1 / 10, \lambda_{T R}=1, R_{\lambda}=r_{\lambda}=2$ and $\tau=10^{-10}$.

\subsection{Constrained optimization with manifold mapping}

If a fast-to-evaluate approximation $\mathbf{k}_{\mathbf{c}}$ of the fine constraint function $\mathbf{k}_{\mathbf{f}}$ is available, the manifold-mapping idea can be extended to a constrained optimization problem in a straightforward way. An additional (affine) mapping $\mathbf{K}: \mathbf{k}_{\mathbf{c}}(\hat{X}) \rightarrow \mathbf{k}_{\mathbf{f}}(\hat{X})$ between the constraint sets, $\mathbf{k}_{\mathbf{c}}(\hat{X})$ and $\mathbf{k}_{\mathbf{f}}(\hat{X})$ is introduced. With $\mathbf{K}$, the point $\mathbf{k}_{\mathbf{c}}\left(\mathbf{x}_{\mathbf{f}}^{*}\right)$ is mapped to $\mathbf{k}_{\mathbf{f}}\left(\mathbf{x}_{\mathbf{f}}^{*}\right)$ and the tangent manifold for $\mathbf{k}_{\mathbf{c}}(\hat{X})$ at $\mathbf{k}_{\mathbf{c}}\left(\mathbf{x}_{\mathbf{f}}^{*}\right)$ to the tangent manifold for $\mathbf{k}_{\mathbf{f}}(\hat{X})$ at $\mathbf{k}_{\mathbf{f}}\left(\mathbf{x}_{\mathbf{f}}^{*}\right)$. Again, the mapping $\mathbf{K}$ is not known a priori and therefore, it has to be approximated iteratively. We indicate in Figure 3 how the MM algorithm has to be modified in order to deal with constraints that are expensive to evaluate (the approximated constraint manifold mapping is denoted by $\mathbf{K}_{k}$ ). The stationary point of the iteration is the right optimum $\mathbf{x}_{\mathbf{f}}^{*}$ since in the limit, the Karush-Kuhn-Tucker conditions (Nocedal and Wright, 1999) hold.

\subsection{Trust-region manifold mapping}

An important requirement for convergence of the MM algorithm is that the matrices $\Delta F$ and $\Delta C$ are sufficiently well-conditioned (Echeverría and Hemker, 2006). In practice it is hard to foresee the effect of this requirement. In the initial phase of the whole optimization process we cannot give any a priori guarantee concerning the condition number associated to the mentioned matrices.

When these matrices are ill-conditioned, the algorithm can take very large steps because of the pseudo-inverse computation. Therefore, in (Hemker and 
Echeverría, 2006) a trust-region strategy (Conn et al., 2000) is introduced in such a way that the undesired effects do no harm and convergence is accelerated. The matrices $\Delta F$ and $\Delta C$ are regularized with the help of a generalized singular value decomposition (GSVD) of the pair $(\Delta F, \Delta C)$.

The GSVD of the pair of matrices $(\Delta F, \Delta C$ ) is (cf. (Golub and van Loan, 1983)) a set of five matrices $U_{\Delta F}, U_{\Delta C}, \Sigma_{\Delta F}, \Sigma_{\Delta C}$ and $V$, such that

$$
\Delta F=U_{\Delta F} \Sigma_{\Delta F} V^{T} \quad \text { and } \quad \Delta C=U_{\Delta C} \Sigma_{\Delta C} V^{T},
$$

with $V$ a regular matrix, $U_{\Delta F}$ and $U_{\Delta C}$ unitary matrices and $\Sigma_{\Delta F}=\operatorname{diag}\left(\sigma_{1}^{\Delta F}, \cdots, \sigma_{n}^{\Delta F}\right) \quad$ and $\quad \Sigma_{\Delta C}=\operatorname{diag}\left(\sigma_{1}^{\Delta C}, \cdots, \sigma_{n}^{\Delta C}\right)$ diagonal matrices with nonnegative elements. The matrix $\Delta C \Delta F^{\dagger}$ in the MM algorithm can now be written as

$$
\begin{aligned}
\Delta C \Delta F^{\dagger} & =U_{\Delta C} \Sigma_{\Delta C} V^{T}\left(V^{T}\right)^{-1} \Sigma_{\Delta F}^{\dagger} U_{\Delta F}^{T}=U_{\Delta C} \operatorname{diag}\left(\sigma_{i}^{\Delta C} / \sigma_{i}^{\Delta F}\right) U_{\Delta F}^{T} \\
& \approx U_{\Delta C} \operatorname{diag}\left(\frac{\sigma_{i}^{\Delta C}+\lambda \sigma_{1}^{\Delta C}}{\sigma_{i}^{\Delta F}+\lambda \sigma_{1}^{\Delta F}}\right) U_{\Delta F}^{T}, \quad \text { with } \lambda \geq 0
\end{aligned}
$$

Regularization is introduced by taking $\lambda>0$. The choice of the parameter $\lambda$ in the trust-region MM algorithm (TRMM, Figure 4) is based on the success of the previous iteration steps. If the residual $\left\|\mathbf{f}\left(\mathbf{x}_{k+1}\right)-\mathbf{y}\right\|$ decreases, the value of $\lambda$ is divided by $r_{\lambda}$, otherwise it is multiplied by $R_{\lambda}$. In practice, $\lambda$ is never reduced below a tolerance $\tau$. The values suggested for a few constants in the algorithm are based on heuristics. Further, the scheme contains an (optional) damping parameter $\delta \geq 0$. For strongly nonlinear problems it can stabilize the convergence process at the expense of a few additional function evaluations (Hemker and Echeverría, 2006).

\section{Optimization Experiments}

The two design problems in this section have practical relevance. The first problem has an expensive constraint function and thus, it is suitable for the manifold-mapping variant presented in Section 3.1. The second one is the wellknown TEAM problem 25 for which we will see that the trust-region strategy in Section 3.2 makes a significant improvement on the original manifold-mapping algorithm.

Two optimization methods are used for the function argmin in the schemes introduced in this paper: sequential quadratic programming (SQP) (Nocedal and Wright, 1999) and differential evolution (DE) (Storn and Price, 1995). We use SQP and DE for local and global minimization purposes respectively.

\subsection{A class-E power amplifier}

In many electronic circuits, the proper device operation is specified by a number of constraints that very often are expensive to evaluate (for example, when the voltage in a node should be larger than a certain threshold).

Class-E power amplifiers (Sokal and Sokal, 1975; Ramos, 2005) are widely used in the radio frequency and microwave field because of their high efficiency. In the amplifier in Figure 5 we see a transistor $\mathrm{M}_{1}$ followed by a resonant filter 


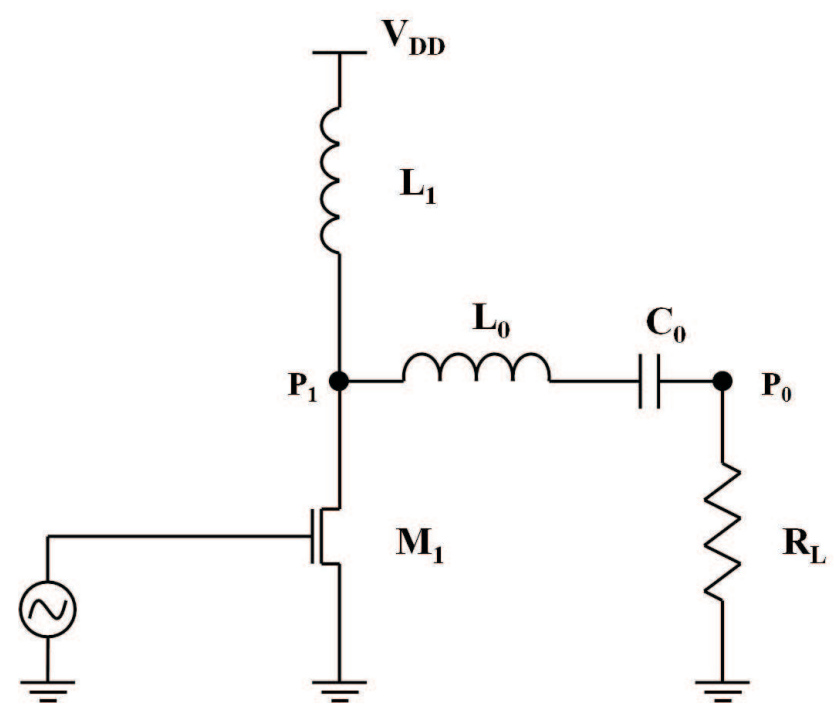

Figure 5: Class-E power amplifier. It consists of a transistor (CMOS) $\mathrm{M}_{1}$, two inductors $\mathrm{L}_{0}$ and $\mathrm{L}_{1}$, one capacitor $\mathrm{C}_{0}$ and a load resistor $\mathrm{R}_{\mathrm{L}}$.

$\mathrm{L}_{0}-\mathrm{C}_{0}$. The transistor acts as a switch and drives the current through the inductor $\mathrm{L}_{1}$.

The design problem consists in the minimization of the supplied power (i.e., the model response is a scalar and $\mathbf{y}=0$ ). The constraints are defined over $\mathbb{R}^{5}$ and they ensure that the circuit is an amplifier that delivers an output power larger than a minimum value. We need to compute the current through $\mathrm{L}_{1}$ in order to obtain the supplied power (the supplied voltage $\mathrm{V}_{\mathrm{DD}}$ is constant and equal to $2 \mathrm{~V}$ ). The constraints are based on the voltages in nodes $\mathrm{P}_{0}$ and $\mathrm{P}_{1}$. The nature of the amplifier implies in all cases expensive transient simulations. The design variable $\mathbf{x}$ is defined over $\mathbb{R}^{5}: x_{1}$ is the internal drawn channel width of the transistor, $x_{2}$ and $x_{3}$ the inductances $\mathrm{L}_{1}$ and $\mathrm{L}_{0}$ respectively, $x_{4}$ the capacitance $\mathrm{C}_{0}$ and $x_{5}$ the load resistance $\mathrm{R}_{\mathrm{L}}$. For a more complete problem description see (Ramos, 2005).

The fine model response and constraint function, $\mathbf{f}$ and $\mathbf{k}_{\mathbf{f}}$, result from a transient simulation where the input wave is a square signal of 50 cycles with a time resolution of 1000 samples per cycle ${ }^{1}$. The cost function is $F(\mathbf{x})=|\mathbf{f}(\mathbf{x})-\mathbf{y}|$. The coarse model and constraint function, $\mathbf{c}$ and $\mathbf{k}_{\mathbf{c}}$, use only 5 cycles with 100 samples per cycle. In both cases, the backward Euler scheme is used for the integration of the corresponding differential equation. In the second situation the computation of the model and the constraint function is 100 times faster compared to the first one. In Figure 6 we show the transient voltages (last cycle) in node $\mathrm{P}_{1}$ for the fine and the coarse simulations with $\mathbf{x}=\mathbf{x}_{\mathbf{f}}^{*}$. Though the differences may seem small, $\mathbf{x}_{\mathbf{f}}^{*}$ is only feasible for the fine constraints.

The manifold-mapping variant for constrained optimization yields the solution $\mathbf{x}=[17530 \mu \mathrm{m}, 3.54 \mathrm{nH}, 1.33 \mathrm{nH}, 63.1 \mathrm{pF}, 3.03 \Omega]$ after 26 equivalent fine transient simulations. (This value is approximately proportional to the total

\footnotetext{
${ }^{1}$ The frequency of operation is $850 \mathrm{MHz}$.
} 


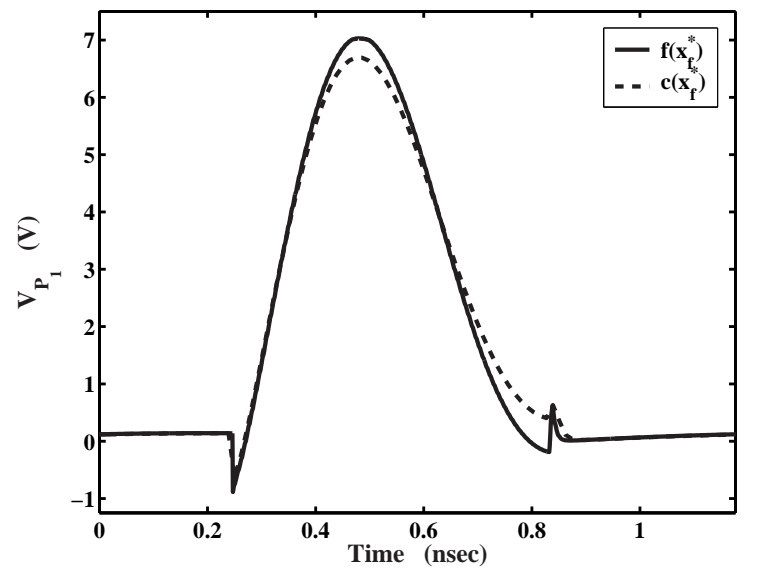

Figure 6: Transient voltages in node $\mathrm{P}_{1}$ for the fine and the coarse simulations when the design variable is the fine model optimum $\mathbf{x}_{\mathbf{f}}^{*}$. The conditions ensuring that the device is an amplifier are based on different features of this waveform.

computing time.) The associated (fine) supplied power is $0.694 \mathrm{~W}$. We use a combination of DE and SQP for the coarse model optimum computation and only SQP for the rest of the minimization processes within MM. The solution given in (Ramos, 2005) is also feasible and shows a supplied power of $0.774 \mathrm{~W}$. But it is computed with DE and therefore, a few thousand simulations are needed. SQP, with the coarse model optimum as initial guess, finds a feasible point (associated supplied power of $0.700 \mathrm{~W}$ ) after 56 equivalent fine transient simulations.

Subsequent optimization runs detect different design solutions with analogous quality. The costs to compute them are also similar to those indicated above. The presence of multiple optima is a clear sign of ill-conditioned model responses and/or constraint functions. The influence of this fact in the performance of the MM scheme has been almost imperceptible. More difficult cases could be dealt with by means of a proper trust-region strategy.

\subsection{A die press (TEAM Problem 25)}

We will study the optimization of a die press with an electromagnet (TEAM Workshop problem 25) (Takahashi et al., 1996). This device (see Figure 7) is used for manufacturing anisotropic permanent magnets. The molds and the pole are made of steel.

The specifications $\mathbf{y}$ are the magnetic flux computed in ten points along the curve e-f (see Figure 7 ) in the cavity; i.e., $\mathbf{y}=\left[B_{x} ; B_{y}\right]$ where $B_{x}$ and $B_{y}$ are the following column vectors

$$
\begin{aligned}
& B_{x}=\left[0.35 \cos \theta_{i}\right]_{i=1, \cdots, 10} \quad(\mathrm{~T}), \\
& B_{y}=\left[0.35 \sin \theta_{i}\right]_{i=1, \cdots, 10}(\mathrm{~T})
\end{aligned}
$$

and $\theta_{i}$ denotes the angle with the $x$-axis. The design variable $\mathbf{x}=\left[x_{1}, x_{2}, x_{3}, x_{4}\right]$ refers to the geometry of the molds. The control space 

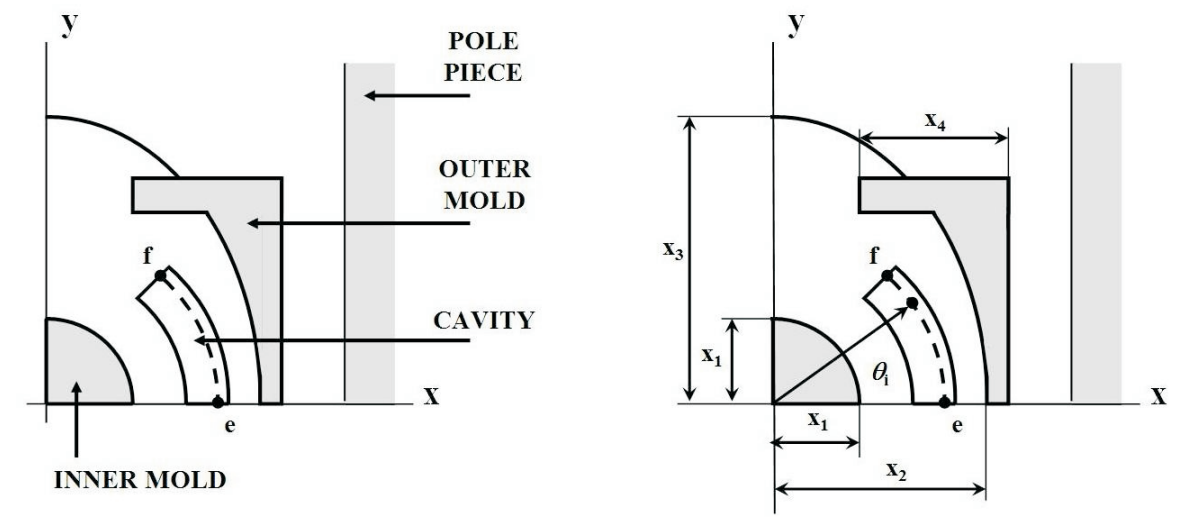

Figure 7: Geometry and design variables of the die press. The die press is symmetric with respect to both axes.

\begin{tabular}{||c|c|c|c|c|c|c||}
\hline & \multicolumn{2}{|c|}{ SQP } & \multicolumn{2}{c|}{ MM } & \multicolumn{2}{c||}{ TRMM } \\
\hline \hline \# iter & \# f evals. & $F(\mathbf{x})$ & \# f evals. & $F(\mathbf{x})$ & \# f evals. & $F(\mathbf{x})$ \\
\hline 1 & 11 & 0.0016 & 1 & 0.0017 & 1 & 0.0018 \\
\hline 2 & 17 & 0.0005 & 2 & 0.0029 & 3 & 0.0013 \\
\hline 3 & 23 & 0.0005 & 3 & 0.0007 & 4 & 0.0007 \\
\hline 4 & 29 & 0.0005 & 4 & 0.0008 & 7 & 0.0007 \\
\hline 5 & 35 & 0.0005 & 5 & 0.0013 & 8 & 0.0004 \\
\hline
\end{tabular}

Table 1: Iteration history for SQP, MM and TRMM in the die-press optimization problem. SQP was applied with the coarse model optimum $\mathbf{x}_{\mathbf{c}}^{*}$ as initial guess. The field \# f evals. denotes the cumulative number of equivalent fine model evaluations (approximately proportional to computing time). The field $F(\mathbf{x})$ indicates the cost function.

is a polytope in $\mathbb{R}^{4}$ (inexpensive constraint function). The complete problem description can be found in (Takahashi et al., 1996).

The fine model $\mathbf{f}$ is based on second order triangular finite elements with four levels of adaptive refinement (this yields around 120000 degrees of freedom). The cost function is $F(\mathbf{x})=\|\mathbf{f}(\mathbf{x})-\mathbf{y}\|_{2}^{2}$. The coarse model $\mathbf{c}$ is built as a least squares quadratic approximation of sixteen finite element solutions with a linearized characteristic of the molds and the pole. The sixteen points in the approximation are vertices of the polytope $X$. The discretizations used there are based on very coarse grids (with less than 1000 degrees of freedom). The construction and evaluation of the coarse model has a negligible computational cost when compared with that of the fine model.

Table 1 shows the die press optimization results. We observe that MM after the fourth iteration seems to move away from the optimum. This diverging tendency grows during the next iterations. The solution $\mathbf{x}=[7.14,13.70,14.00,14.33](\mathrm{mm})$ is computed by TRMM in five iterations (eight equivalent fine model evaluations). The associated cost function value is 0.0004 and the quality of the solution is acceptable for practical purposes. We 
use DE for the coarse model optimum computation and for every minimization process within the manifold-mapping approach. TRMM performs much more efficient than SQP, which was applied with the coarse model optimum as initial guess.

\section{Conclusions}

Manifold mapping is a multi-level surrogate-based optimization strategy aimed at very time-consuming design problems. In this paper we have presented two new variants of the original manifold-mapping algorithm. The first one is useful when the optimization constraints are given by functions that are very expensive to compute. The second one incorporates a trust-region strategy that yields a much more robust scheme. Both variants perform efficiently in solving practical design problems from electromagnetics.

\section{Acknowledgement}

The author would like to thank P.W. Hemker for his kind and detailed feedback to this manuscript and J. ter Maten, C. Lin and J. Ramos for their help concerning the class-E power amplifier problem. This research is supported by the Dutch Ministry of Economical Affairs within the project IOP-EMVT 02201.

\section{References}

J.W. Bandler, R.M. Biernacki, S.H. Chen, P.A. Grobelny, and R.H. Hemmers. Space Mapping Technique for Electromagnetic Optimization. IEEE Transactions on Microwave Theory and Techniques, 42(12):2536-2544, 1994.

J.W. Bandler, Q.S. Cheng, A.S. Dakroury, A.S. Mohamed, M.H. Bakr, K. Madsen, and J. Søndergaard. Space Mapping: The State of the Art. IEEE Transactions on Microwave Theory and Techniques, 52(1):337-361, 2004.

A.R. Conn, N. Gould, and Ph.L. Toint. Trust-Region Methods. MPS/SIAM Series on Optimization. SIAM, Philadelphia, US, 2000.

D. Echeverría and P.W. Hemker. Space Mapping and Defect Correction. Comp. Methods in Appl. Math., 5(2):107-136, 2005.

D. Echeverría and P.W. Hemker. On the Manifold-Mapping Optimization Technique. Technical Report MAS-R0612, Centrum voor Wiskunde en Informatica (CWI), Amsterdam, The Netherlands, June 2006.

D. Echeverría, D. Lahaye, L. Encica, and P.W. Hemker. Optimisation in Electromagnetics with the Space-Mapping Technique. COMPEL The International Journal for Computation and Mathematics in Electrical and Electronic Engineering, 24(3):952-966, 2005.

D. Echeverría, D. Lahaye, L. Encica, E.A. Lomonova, P.W. Hemker, and A.J.A. Vandenput. Manifold-Mapping Optimization Applied to Linear Actuator Design. IEEE Transactions on Magnetics, 42(2):1183-1186, 2006. 
G.H. Golub and C.F. van Loan. Matrix Computations. North Oxford Academic, Oxford, England, 1983.

P.W. Hemker and D. Echeverría. A Trust-Region Strategy for ManifoldMapping Optimization. Technical Report MAS-E0618, Centrum voor Wiskunde en Informatica (CWI), Amsterdam, The Netherlands, August 2006.

J. Nocedal and S.J. Wright. Numerical Optimization. Springer Series in Operations Research. Springer, 1999.

J. Ramos. CMOS Operational and RF Power Amplifiers for Mobile Communications. PhD thesis, K.U. Leuven, Belgium, March 2005.

N.O. Sokal and A.D. Sokal. Class E: A New Class of High-Efficiency Tuned Single-Ended Switching Power Amplifiers. Journal of Solid-State Circuits, 10 (3):168-176, June 1975.

R. Storn and K. Price. Differential Evolution - A Simple and Efficient Adaptive Scheme for Global Optimization Over Continuous Spaces. Technical Report TR-95-012, International Computer Science Institute (ICSI), March 1995.

N. Takahashi, K. Ebihara, K. Yoshida, T. Nakata, K. Ohashi, and K. Miyata. Investigation of Simulated Annealing Method and its Application to Optimal Design of Die Mold for Orientation of Magnetic Powder. IEEE Transactions on Magnetics, 32(3):1210-1213, May 1996. 